\title{
A Smart Machine Vision based Inspection System
}

\author{
Dr. Santosh Kumar Sahoo \\ Assoc. Professor, CVR College of Engineering/EIE Department, Hyderabad, India \\ Email: santosh.kr.sahoo@gmail.com
}

\begin{abstract}
This article proposes a unique optimization like Adaptive Cuckoo Search $\left(\mathrm{A}_{\mathrm{d}} \mathrm{CS}\right)$ algorithm followed by an Intrinsic Discriminant Analysis (IDA) to design a smart intelligent object classifier for inspection of defective object like bottle in a manufacturing unit. By using this methodology, the response time is very faster than the other techniques. The projected scheme is authenticated using different benchmark test functions and in the next part of the article proposes an efficient recognition algorithm for identification of bottle by using $A_{d} C S$, Principal Component Analysis (PCA) and IDA. Due to this the proposed algorithms terms as PCA+IDA for dimension reduction and $A_{d} C S-I D A$ for classification or identification of defective bottles. The analyzed response obtained from by an application of $\mathrm{A}_{d} \mathrm{CS}$ algorithm followed by IDA and compared to other algorithm like Least-SquareSupport-Vector-Machine (LSSVM) along with Linear Kernel Radial-Basis-Function (RBF) to the proposed model, the earlier applied scheme reveals the remarkable performance
\end{abstract}

Index Terms: Adaptive Cuckoo Search $\left(\mathrm{A}_{d} \mathrm{CS}\right)$ algorithm, Intrinsic Discriminant Analysis (IDA), Principal Component Analysis (PCA), intelligent object classifier, least square Support Vector Machine (LSVM), Smart inspection.

\section{INTRODUCTION}

Due to rapid growth of industry to meet the society's requirement it is necessary for maintaining a quality product. In order to achieve this, most of the manufacturing unit follow the automation scheme as a result a finished quality product can be delivered to an end user within an optimum interval. So, in most of the automation, an image processing scheme is used to facilitate the model more efficient to recognize the object of interest in a smooth manner. The proposed model uses an artificial intelligent scheme for recognition of defective bottle in a manufacturing unit where the different algorithm and techniques are used to validate the suggested plan. Before object identification it is highly necessary to reduce the dimension of the captured image by an application of different linear tools such as PCA, LDA as well as some nonlinear tools like Artificial neural network scheme (ANN), isometric mapping, locally linear embedding and Laplacian Eigen maps etc. But apart from this the linear tools are the best choice because the nonlinear tools required heavy computational work for different parameter tuning and lack of handling capacity for testing data as compared to linear one. The PCA projects the high dimension data into a set of basis function to get a squeezed demo of the original data as a result dimension reduction is achieved and it mainly reflects the Eigen faces of the detected image features. Likewise, LDA reflects the features as a Fisher faces in which the data points of different classes are mapped with an optimum gap or distance. The IDA method is used to maximize the uniqueness dissimilarities in the meantime the intrapersonal difference is minimized. Again, the selection procedure for optimal Eigen vector is inaccurate. So, to overcome this problematic condition the PCA + IDA algorithm remains used for increasing the performance level then this performance is compared with the other identification techniques like $\mathrm{K}^{\mathrm{TH}} \mathrm{NN}$, ANN, SVM and LSSVM method. Finally, it is concluded that the projected scheme be able to classify impaired bottle promptly as well as accurately. Therefore, the curative exploit determination be continuing on a crucial stage of manufacturing progression so that the ruin quality risk be adjusted.

Nouri et. al [1] examined a classification model for recognize a printed Arabic name using density weight and zigzag techniques. The proposed model was validated using KNN and support vector machine classifier. K Tafi et.al [2] described a breast cancer analysis scheme established on wavelet exploration and neural networks. Jayachandran et.al [3] explained about a PC aided judgment of cancer in brain MRI pictures by wavelet techniques and an Ada-Boost classifier. Nayak et.al [4] explained about a time efficient clustering algorithm for gray scale image segmentation and evaluated different clustering algorithms. Gharehchopogh et.al [5] described a novel approach for edge detection of an image by using cellular learning automata (CLA) techniques. Dixit et.al [6] demonstrated an Indian sign language recognition system using a vision approach where a multi class support vector machine algorithm was used. Dubey et.al [7] explained about different fruit disease identification by using image processing scheme like $\mathrm{K}$-means clustering and multi class support vector machine classifier and their proposed model claimed an accuracy of about 93\%. Win et.al [8] implemented a structural analysis-based feature extraction technique for optical character recognition system for Myanmar printed document. Anitha et.al [9] explored a cross breed hereditary procedure grounded fuzzy tactic aimed at retinal image grouping. Krol et.al.[10] implemented a real time automatic mechanism aimed at image and speech acknowledgement centered on neural network where a Neuro car-based system and Neuro scope were used to validate the proposed model. Santo.et.al [11] described different replicated forging schemes. Yaremchuk et.al [12] examined the classification of musical cords by means of artificial neural network. Xu. Et.al [13] demonstrated about an iris acknowledgement scheme by means of Intersecting Cortical Model (ICM) neural network. During investigation the captured image was processed and analyzed by ICM neural network. Xing 
at.al [14] described about three-dimensional object classification based on volumetric parts in which super quadric based Geon description was implemented to representing the volumetric elements of 3D object. Tim et.al [15] examined the firmness efficiency of joint photographic three-dimensional medical images.

\section{Proposed Algorithm}

This proposed optimization algorithm uses an Adaptive Cuckoo Search (AdCS) algorithm followed by an Intrinsic Discriminant Analysis (IDA) to design a smart intelligent object classifier for inspection of defective object like bottle in a manufacturing unit.

\section{A. BASICS ON WAVELET TRANSFORM, PCA,IDA AND}

\section{CUCKOO SEARCH ALGORITHMS}

\section{Wavelet Transform}

Object identification or inspection deals with the high dimensionality of image space under consideration. So a method termed as wavelet transform can be used to decompose the data without gaps or overlap. Considering an image of a bottle as shown in Figure 1 and corresponding segmented picture meant for faulty after preprocessing be presented in Figure 2 .

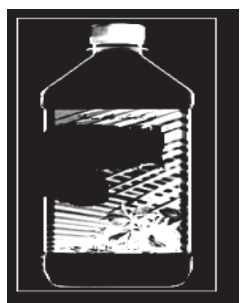

Figure 1. Defective bottle's segmented image

$$
\left[\begin{array}{ccc}
-1 & -2 & -1 \\
0 & 0 & 0 \\
1 & 2 & 1
\end{array}\right]
$$

Figure 2. The mask gradient value

At this moment the wavelet constants for corresponding image are stated as[6] in equation-1 \& 2:

$$
\begin{aligned}
& \mathrm{w}_{\psi}\left(I_{0}, L, K\right)=\frac{1}{\sqrt{1 \times \mathrm{k}}} \sum_{\mathrm{u}=0}^{1-1} \sum_{\mathrm{v}=0}^{\mathrm{k}-1} \mathrm{~F}(u, v) \varphi_{\mathrm{I}_{0} \mathrm{~L}, \mathrm{~K}}(U, V) \\
& w_{\psi}^{I}(I, L, K)=\frac{1}{\sqrt{l \times k}} \sum_{u=0}^{l-1} \sum_{v=0}^{k-1} F(u, v) \phi_{I, L, K}^{I}(U, V)
\end{aligned}
$$

Where $\varphi^{\mathrm{I}}{ }_{\mathrm{I}, \mathrm{L}, \mathrm{K}} \quad(\mathrm{U}, \mathrm{V})=2^{J / 2} \varphi\left(P^{J} U-L, P^{J} V-K\right)$ and

$$
\phi_{J_{0} M N}(\mathrm{U}, \mathrm{V})=2^{I / 2} \varphi\left(P^{J} U-L, P^{J} V-K\right)
$$

Here $P$ value is considered as 2.At present the Energy anticipated totally in vertical, horizontal and diagonal directions as per Figure 3 [5]. As three level of decomposition remains cast-off accordingly the Energy for each wavelet constants are [4] in equation 3. $e_{c l}=\frac{1}{l x l} \sum_{I=0}^{l} \sum_{L=0}^{l}\left(w_{c, l, I, L}\right)^{2}$

\begin{tabular}{|c|c|c|c|}
\hline $\begin{array}{l}\text { Approxim } \\
\text { ate } \\
\text { Significance } \\
\text { of } \\
\text { pixel(3) }\end{array}$ & $\begin{array}{c}\text { Horizontal } \\
\text { meticulous } \\
\text { significance } \\
\text { of pixel(3) }\end{array}$ & \multirow{2}{*}{$\begin{array}{l}\text { Horizontal } \\
\text { meticulous } \\
\text { significance } \\
\text { of pixel(2) }\end{array}$} & \multirow{2}{*}{$\begin{array}{c}\text { Horizontal } \\
\text { meticulous } \\
\text { significance } \\
\text { pixel(1) }\end{array}$} \\
\hline $\begin{array}{c}\text { Vertical } \\
\text { meticulous } \\
\text { significance } \\
\text { of pixel(3) }\end{array}$ & $\begin{array}{c}\text { Diagonal } \\
\text { Meticulous } \\
\text { significance } \\
\text { of pixel(3) }\end{array}$ & & \\
\hline \multicolumn{2}{|c|}{$\begin{array}{c}\text { Vertical } \\
\text { meticulous } \\
\text { significance of } \\
\text { pixel(2) }\end{array}$} & $\begin{array}{l}\text { Diagonal } \\
\text { Meticulous } \\
\text { gnificance of } \\
\text { pixel(2) }\end{array}$ & \multirow{2}{*}{$\begin{array}{l}\text { Diagonal } \\
\text { Meticulous } \\
\text { significance } \\
\text { of pixel(1) }\end{array}$} \\
\hline \multicolumn{3}{|c|}{$\begin{array}{l}\text { Vertical meticulous significance of } \\
\operatorname{pixel(1)}\end{array}$} & \\
\hline
\end{tabular}

Where $w_{C, l, I, L}$ equal to wavelet constant at $[\mathrm{I}, \mathrm{L}]$ location [1-6].

Figure 3: A three level Wavelet transform structure

\section{PCA}

Similarly in PCA a best prediction hatchets from a composite strew of expected samples as a result the total scatter matrix is expressed as [7] in equation-4

$$
M_{S}=\sum_{J=1}^{K} \sum_{i=1}^{L_{i}}\left(X_{j}^{J}-\mu\right)\left(X_{j}^{J}-\mu\right)^{T}
$$

Where $\mu=\frac{1}{L} \sum_{J=1}^{K} \sum_{i=1}^{L_{i}}\left(X_{j}^{J}\right)$ the mean value and the PCA objective function is set by $O_{P C A}=\stackrel{\operatorname{Arg} \max }{O}\left|O^{T} M_{S} O\right|$. Hence the optimum projections of PCA are $\mathrm{O}_{1}, \mathrm{O}_{2}, \mathrm{O}_{3} \ldots \mathrm{O}_{n}$ which basically orthogonal eigenvectors are of $M_{S}$.

IDA: Likewise the IDA is a linear projection based approach and based on individuality difference. It minimizes the individuality difference. The IDA based features for Object recognition are created as intrinsic objects. Let the a ' $n$ ' image sample of ' $y$ ' different classes $\left(\mathrm{C}_{1}, \mathrm{C}_{1} \ldots \ldots . . . \mathrm{Cy}\right)$ and each class has $\mathrm{n}_{\mathrm{i}}$ numbers of samples in class $\mathrm{C}_{\mathrm{i}}$ and $n=\sum_{i=1}^{y} n_{i}$. Again each image be presented as $\mathrm{p} \times \mathrm{q}$ matrix and modeled as $\mathrm{d}$ dimensional $(\mathrm{d}=\mathrm{p} \times \mathrm{q})$ vector formed through a two dimensional pixel array. As a result a set of points presented in equation-5, $6,7,8,9,10,11$, and 12 $\left(\begin{array}{rrrr}X^{a}, \ldots \ldots . X^{a} & \ldots \ldots X^{y} \ldots \ldots . . X^{y} \\ 1 & n_{1} & 1 & n_{y}\end{array}\right)$ 
where $X_{J}^{I}$ is the $J^{\text {th }}$ sample in $I^{\text {th }}$ Class are obtained. Now gathering all the points in to a single matrix $\mathrm{x}$ of size $\mathrm{d} \times \mathrm{n}$ as[8] $x^{J}=\left[X_{1}^{J}, X_{2}^{J}, \ldots \ldots X_{n_{J}}^{J}\right] \in \mathfrak{R}^{d \times n_{J}}$

Assuming a matrix which represents the $\mathrm{J}^{\text {th }}$ class of images as

$x^{J}=\left[X_{1}^{J}, X_{2}^{J}, \ldots \ldots X_{n J}^{J}\right] \in \mathfrak{R}^{d \times n_{J}}$

, then the ' $\mathrm{x}$ ' be able to voice as [9]

$\mathrm{x}=\left[X^{1}, X^{2}, \ldots \ldots X^{y}\right]$.

If a matrix $M_{T}$ is express as [10]-[11]

$M_{T}=\left[X_{1}^{1}-\mu, X_{2}^{1}-\mu, \ldots \ldots . X_{n_{y}}^{y}-\mu\right] \in \mathfrak{R}^{d \times n}$

Then the singular value decomposition (SVD) of $\mathrm{M}_{\mathrm{T}}$ is expressed as [12].

$M_{T}=U \sum V^{T}$

Where $\quad U=\left[U_{r}, \overline{U_{r}}\right] \quad, \quad U r \in \Re^{d \times n} J$, $\overline{U r} \in \mathfrak{R}^{d(d-r)}, \quad V=\left\lfloor V_{r}, \overline{V_{r}}\right\rfloor, \mathrm{V}_{\mathrm{r}} \in \mathfrak{R}^{\mathrm{n} \times \mathrm{r}}$

$\overline{\mathrm{V}_{\mathrm{r}}} \in \mathfrak{R}^{\mathrm{n} \times(n-\mathrm{r})} \quad, \quad \sum=\left[\begin{array}{rr}\sum_{1} & 0 \\ 0 & 0\end{array}\right]$ is a non-singular and $\mathrm{R}=$

Rank .

Then after for the Jth class matrix $M_{T}^{J}=\left[X_{1}^{J}-\mu, X_{2}^{J}-\mu, \ldots \ldots . . X_{n_{J}}^{J}-\mu\right] \in \Re{ }^{d \times n} J$, the SVD of $\mathrm{M}_{\mathrm{T}}^{\mathrm{J}}$ as [13] $M_{T}^{J}=U^{J}{ }^{J} V^{J T}$

Where

$U^{J}=\left[\begin{array}{cc}U^{J} & \overline{U^{J}} \\ r^{J}\end{array}\right]$

$U_{r^{J}}^{J} \in \mathfrak{R}^{d \times r^{J}}, \overline{U_{r^{J}}^{J}} \in \mathfrak{R}^{d \times\left(d-r^{J}\right)}, r^{J}=\operatorname{rank}\left(M_{T}^{J}\right)$

An IDA can maximize the difference between the separate classes of images while minimizing the difference within the similar classes of images. Or in other hand IDA tries to make best use of the fraction of the individuality deference distributed Matrix. Now the objective function of IDA is expressed as [14]-[15]

$O_{I D A}=\underset{O}{\arg \max } \frac{\left|O^{T} M_{C} O\right|}{\left|O^{T} M_{I} O\right|}$

The optimal transformation vector is taken from $\mathrm{q}$ number of eigenvalues such that $\lambda_{1} \geq \lambda_{2} \geq \ldots \lambda_{q}$ this can be achieved by solving the eigenvalue problem $\mathrm{M}_{\mathrm{C}} \mathrm{p}=\lambda \mathrm{M}_{\mathrm{I}} \mathrm{p}$

\section{Cuckoo search Algorithm:}

This method considers the communal thoughtful ability of a Cuckoo bird. Generally, the Cuckoo leaves an egg in the nest of the crow's nest. The crow may be recognizing the cuckoo's egg with a probability of then the crow through the eggs from the nest or abandons the next to form a new nest. So now every single egg in the host shell signifies a explanation and to form this one towards a mathematical model the following assumptions are taken as: Available host nests are fixed, Assuming every single Cuckoo be able to lays only single egg at a phase by the side of the randomly selected host net, the finest nets by utmost eminence eggs will transport forward for subsequent generation. Suppose is the present hunt space of Cuckoo $\mathrm{J}$ for $(\mathrm{J}=1,2,3, \ldots . \mathrm{n})$ at time $\mathrm{t}$ represented as $x_{J}(t)=\left(X_{J}^{1}, X_{J}^{2}, \ldots \ldots X_{J}^{d}\right)$ in the 'd' dimension problem at that time the innovative key $x_{J}(t+1)$ for the next generation of interval $(t+1)$ be able to conveyed by way of $x_{J}(t+1)=x_{J}(t)+\alpha \oplus \operatorname{levy}(\lambda)$. Where $\alpha=$ stair dimension and usually this value should be taken as 1 , the $\oplus$ symbol signifies multiplication entry wise and $\operatorname{levy}(\lambda)$ reflects unsystematic walk through levy flight for distribution. Now $\operatorname{levy}(\lambda)=\frac{u}{|v|^{\frac{1}{\lambda-1}}}$.

Considering the present generation ' $\mathrm{t}$ ' and predetermined maximum lifetime iteration $\mathrm{t}_{\mathrm{Max}}$, the Cuckoo at initial generation $(\mathrm{t}=1)$ can be reflected as $x_{J}^{d}(t=1)=\operatorname{rand} x\left(\right.$ Upper $^{d}-$ Lower $\left.^{d}\right)+$ Lower $^{d}$

Where Upper $^{d}$ and Lower ${ }^{d}$ are termed as upper and lower boundaries of the search space.

As the Cuckoo search algorithm uses the levy steps to discover the search space which is taken from levy distribution. Here in proposed algorithm the Cuckoo search algorithm is modeled without using levy distribution. Hence the step size or stair dimension [7] here considered remains proportionate to suitability of the singular shell in hunt galaxy for present peer group. Now this algorithm gives the step size

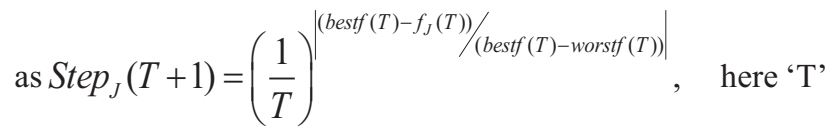
signifies the peer group of the Cuckoo exploration , $\mathrm{f}_{1}(T)=$ fitness cost for $\mathrm{J}^{\text {th }}$ nest , best $\mathrm{f}_{1}(T)$ is finest fitness cost in peer group ' $\mathrm{T}$ ' then worst $f_{1}(T)=$ worst fitness cost in the peer group ' $\mathrm{T}$ '. 


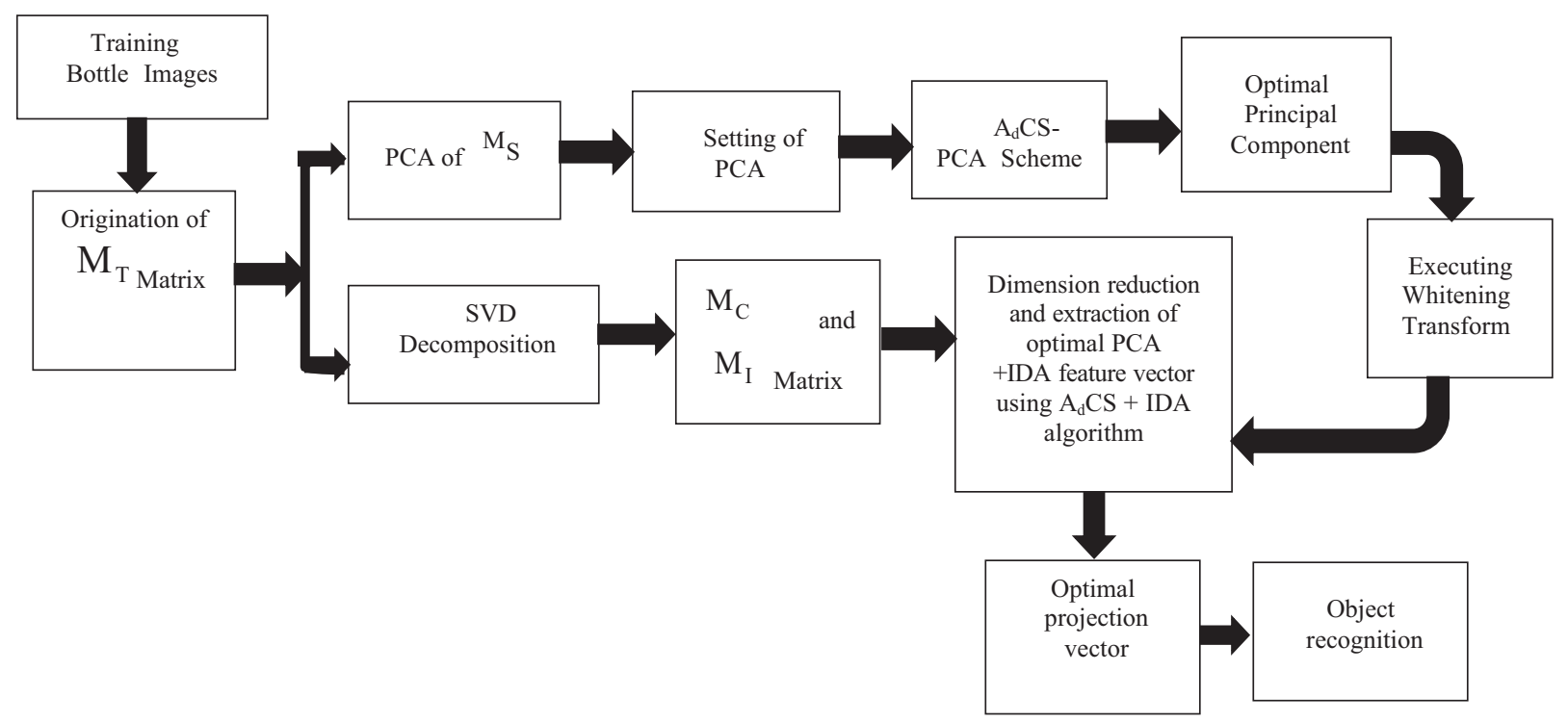

Figure 4. Bottle inspection using PCA plus IDA Scheme

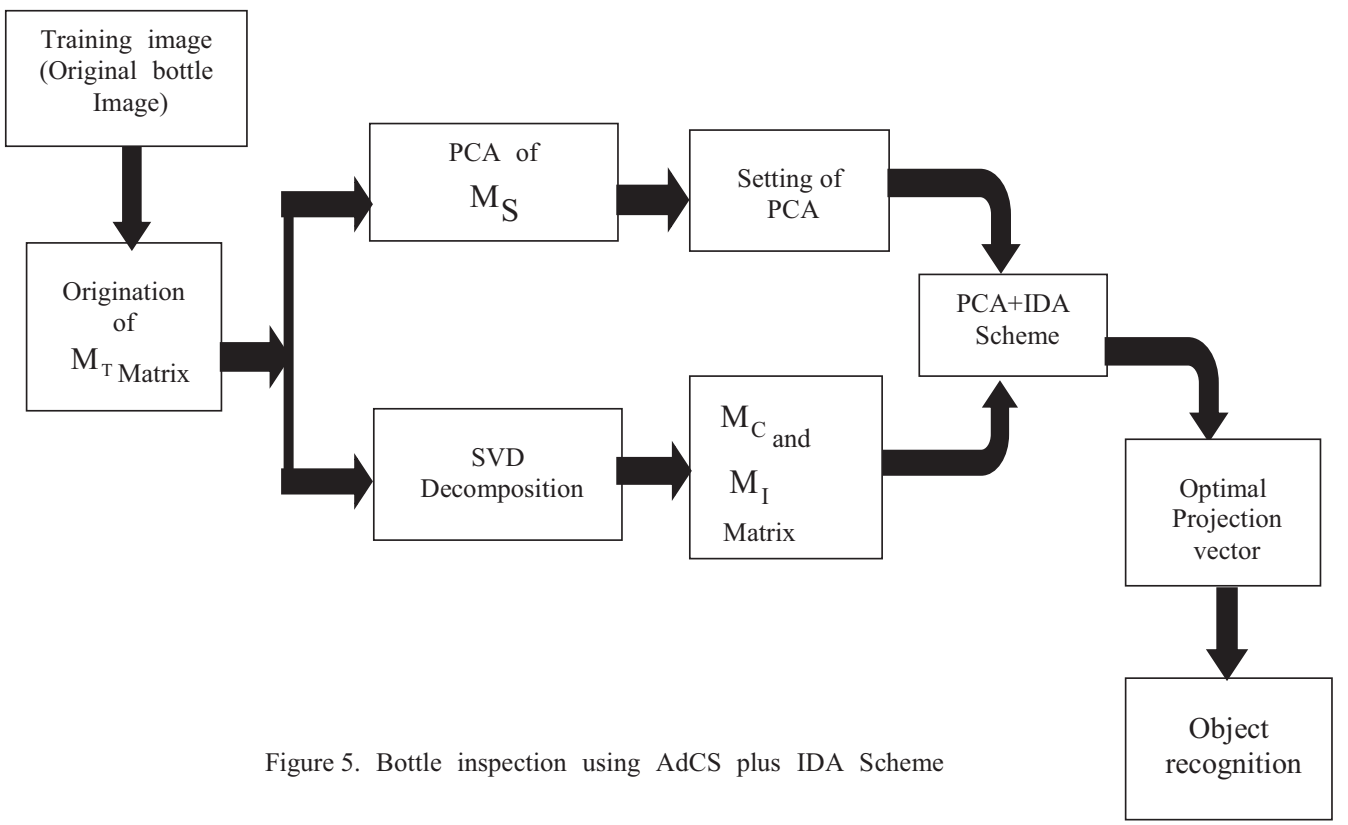

TABLE I.

Bottle InSPECtion Performance

\begin{tabular}{|c|c|c|c|c|c|c|c|c|c|c|c|c|}
\hline $\begin{array}{l}\text { No. of } \\
\text { Training } \\
\text { Image }\end{array}$ & Algorithm & $\begin{array}{l}\text { Run } \\
\text { Time } \\
(\mathrm{Sec}) \\
\end{array}$ & $\begin{array}{c}\text { Rank } \\
1 \text { in } \\
\%\end{array}$ & $\begin{array}{c}\text { Rank } \\
2 \text { in } \\
\%\end{array}$ & $\begin{array}{c}\text { Rank } \\
\text { 3in } \\
\%\end{array}$ & $\begin{array}{c}\text { Rank } 4 \\
\text { in \% }\end{array}$ & $\begin{array}{c}\text { Rank } \\
5 \text { in } \\
\%\end{array}$ & $\begin{array}{c}\text { Rank } 6 \\
\text { in } \%\end{array}$ & $\begin{array}{c}\text { Rank } \\
7 \text { in } \\
\%\end{array}$ & $\begin{array}{c}\text { Rank } 8 \\
\text { in \% }\end{array}$ & $\begin{array}{c}\text { Rank } \\
9 \text { in } \\
\%\end{array}$ & $\begin{array}{c}\text { Rank } \\
10 \text { in } \\
\%\end{array}$ \\
\hline \multirow{3}{*}{3 Train } & $A_{d}$ CS-IDA & 8.15 & 79.4 & 83.6 & 86.8 & 92.5 & 93.4 & 93.4 & 94.4 & 98.2 & 98.5 & 98.5 \\
\hline & PCA+IDA & 61.44 & 76 & 81.7 & 84.2 & 91.5 & 92.1 & 93.7 & 94.4 & 96.8 & 96.8 & 96.8 \\
\hline & IDA & & 76 & 76.6 & 79.2 & 89.6 & 89.2 & 89.4 & 92.6 & 92.6 & 94.4 & 94.3 \\
\hline \multirow{3}{*}{4 Train } & $A_{d}$ CS-IDA & 8.05 & 79.2 & 82.6 & 83.8 & 91.4 & 93.4 & 93.8 & 95.4 & 97.4 & 98.4 & 98.4 \\
\hline & $\mathrm{PCA}+\mathrm{IDA}$ & 33.94 & 78.2 & 80.2 & 83.2 & 91.4 & 92.3 & 93.8 & 94.2 & 96.6 & 96.5 & 96.5 \\
\hline & IDA & & 76 & 76.6 & 79.2 & 89.6 & 89.2 & 89.4 & 92.6 & 92.6 & 94.4 & 94.4 \\
\hline \multirow{3}{*}{5 Train } & $A_{d}$ CS-IDA & 7.11 & 89.6 & 91.6 & 92.4 & 92.6 & 93.7 & 94.8 & 95.7 & 97.6 & 98.5 & 98.7 \\
\hline & PCA+IDA & 13.6 & 87.2 & 84.2 & 86.4 & 92.3 & 93.4 & 93.9 & 94.6 & 96.7 & 96.8 & 96.8 \\
\hline & IDA & & 84.2 & 84.8 & 86.7 & 88.4 & 90.4 & 91.8 & 92.8 & 93.2 & 94.8 & 94.8 \\
\hline
\end{tabular}


Initially stair dimension is more, while No. of peer group rises then the step size is declines which directs overall finest solution. Now $\mathrm{A}_{\mathrm{d}} \mathrm{CS}$ [27] represented as $x_{J}\left(T_{m}+1\right)=x_{J}\left(T_{m}\right)+$ rand $\times \operatorname{Step}_{J}\left(T_{m}+1\right)$. Assuming $T_{m}=T$

The above expression it is concluded that the $\mathrm{A}_{\mathrm{d}} \mathrm{CS}$ does not required any initial parameter and it is very faster than cuckoo search techniques. The proposed algorithm structure is shown in Figure 4 and 5. The block diagram of proposed PCA+IDA based bottle inspection scheme is shown in Figure 4 where the dimension of the feature space can be reduced to $n-K$. Similarly, Figure 5 represents an $\mathrm{A}_{d} \mathrm{CS}$ based IDA bottle identification scheme in which the both dimension reduction method using PCA+IDA and dimension reduction approach using $\mathrm{A}_{\mathrm{d}} \mathrm{CS}$-PCA along with a whitening procedure to develop a $\mathrm{A}_{d} \mathrm{CS}$-IDA algorithm for bottle inspection.

By considering a set of around 100 test images of bottle including defective and defect free the model is verified by using the proposed algorithm. In this proposed scheme we use $\mathrm{A}_{d} \mathrm{CS}-\mathrm{PCA}$ and PCA+IDA.

\section{Result AnAlysis}

For the bottle inspection where the $\mathrm{A}_{\mathrm{d}} \mathrm{CS}-\mathrm{PCA}$ is used for dimension reduction and PCA+IDA used for getting optimal projection vector. By implementing PCA+IDA which gives a agitation free dimension reduction as a result higher efficiency in inspection. As PCA is agitation free so that a time essential for global optimal value evaluation for the projected procedure remains matched with the IDA as per data shown in table1, where the time required for bottle inspection corresponding to different approach or scheme is presented.

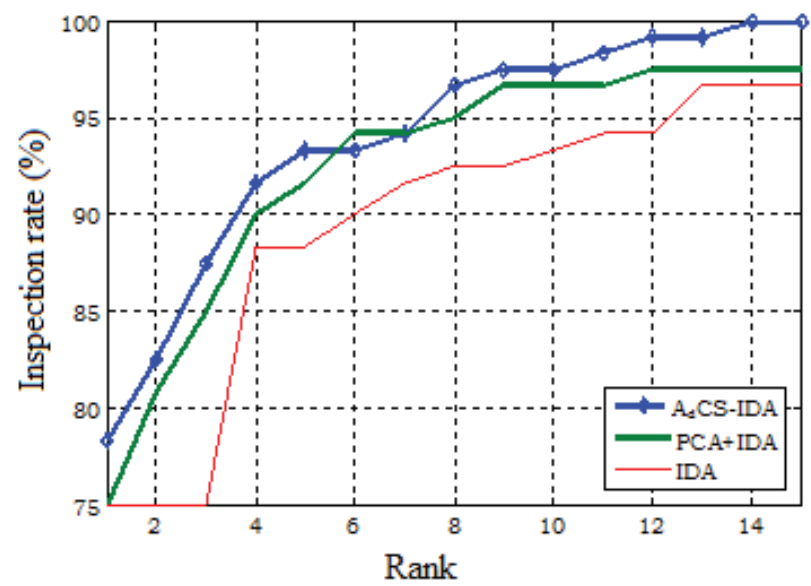

Figure 6 (a). Bottle inspection performance using proposed scheme for a 3 train Image

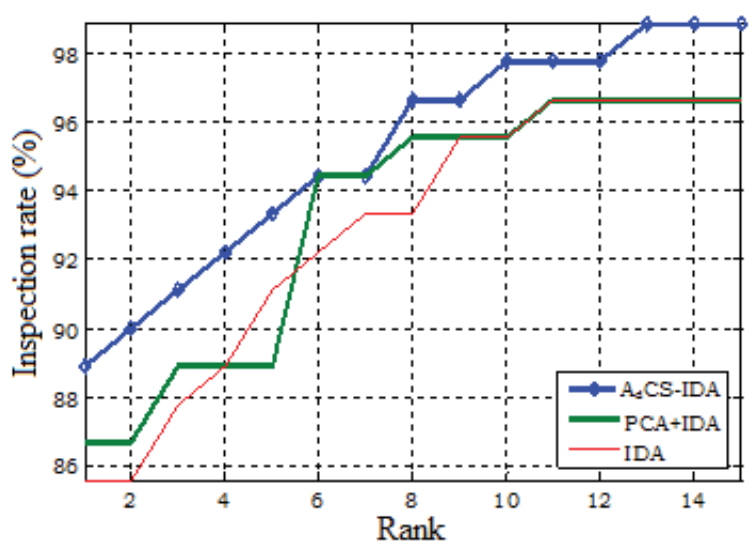

Figure 6 (b). Bottle inspection performance using proposed scheme for a 4 train Image

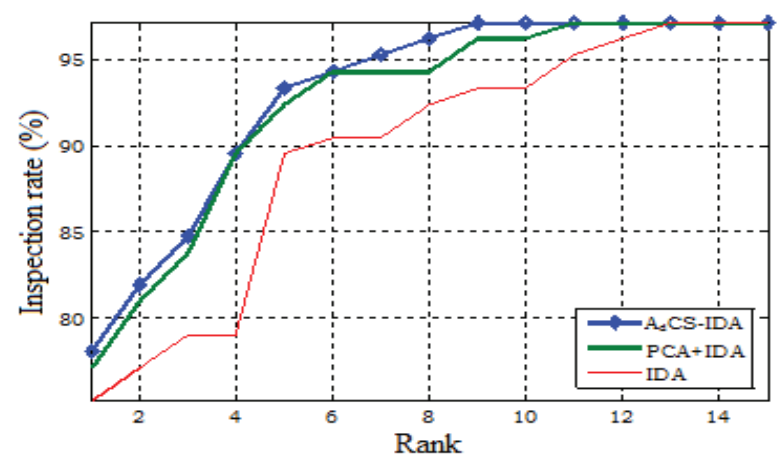

Figure 6(c). Bottle inspection performance using proposed scheme for a 5 train Image

Inspection rate (\%) of different methods on Bottle database at rank 10

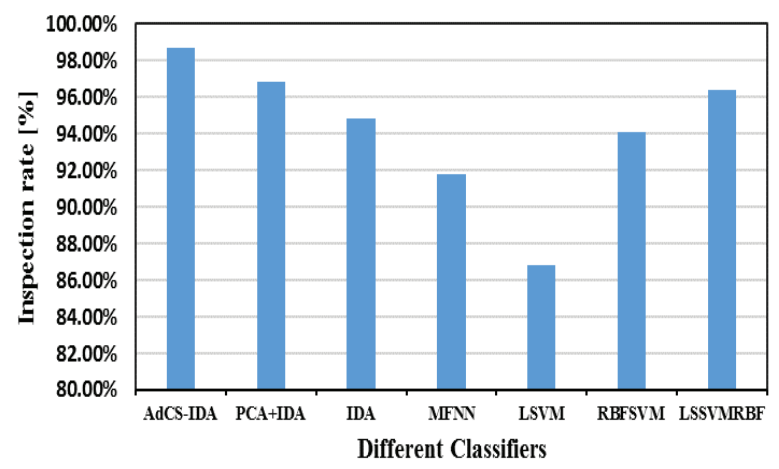

Figure 7. Inspection rate Comparison of different scheme 
TABLE II.

INSPECTION RATE (\%) OF DIFFERENT METHODS ON BOTTLE DATABASE AT RANK10

\begin{tabular}{|c|c|}
\hline Classifiers & Inspection rate (\%) \\
\hline $\mathbf{A}_{\mathbf{d}}$ CS-IDA & $\mathbf{9 8 . 7 \%}$ \\
\hline PCA+IDA & $96.8 \%$ \\
\hline IDA & $94.8 \%$ \\
\hline Multi feed Neural network (MFNN) & $91.78 \%$ \\
\hline LSVM & $86.84 \%$ \\
\hline RBFSVM & $94.06 \%$ \\
\hline LSSVMRBF & $96.35 \%$ \\
\hline \hline
\end{tabular}

From the Table I it is perceived that the anticipated procedure has an improved inspection rate than the additional classical method by considering the different bottle image data base. From Table I and Figure 6(a, b, c) it is concluded that the proposed algorithm has significant improvement over IDA. By comparing two proposed algorithm the $\mathrm{A}_{\mathrm{d}} \mathrm{CS}$-IDA has better inspection rate than PCA+IDA. Again, the proposed $\mathrm{A}_{d} \mathrm{CS}$-IDA consumes more time than other because of it employs the adaptive Cuckoo search techniques. Again, by using different training images for computation it is observed that the computation time is gradually reduced hence it is claimed that the proposed scheme is best suitable for bottle inspection.

\section{CONCLUSIONS}

For Bottle inspection there are different schemes are adopted but the proposed scheme used here for the same task is a best fit and more efficient because of using an adaptive Cuckoo search technique in which the step size can be adaptably decided from the acquaintance of fitness value and current position. The proposed algorithm $\mathrm{A}_{d} \mathrm{CS}$-IDA is used for defective bottle inspection in a manufacturing unit is known as adaptive cuckoo search intrinsic discriminant investigation. The proposed method $\mathrm{A}_{d} \mathrm{CS}-\mathrm{IDA}$ and PCA+IDA are validated with the image data base of different bottles. The different classifiers performance when studied in Table II, concluded that the suggested scheme is a suitable one for inspection of bottle in better way.

\section{REFERENCES}

[1] Nouri !Said, !Fakir !Mohamed. !(2014). !Classification of !printed !Moroccan !town !and !village !names. !Journal of !Information !Technology !Research.Volume-7, !Issue-4, !Pg.111.DOI: !10. !4018 !/ !jitr.2014100101.

[2] K. Tafi, !S. Safi, !M. Fakir. !(2014). !Breast !cancer !diagnosis !system based !on !wavelet !analysis !and !neural !networks, !International !journal of !Computer !vision !and !image !processing, !4(1), !Pg.1-16, !DOI:10.4018/ !ijcvip. !2014 !010101.
[3] Jayachandran !A.,Dhanasekaran !R. !(2014). !Computer !assisted !diagnosis of !tumor ! in !brain !MRI !images !using !wavelet !as !input !to !Ada-boost !classifier. !International !journal of !Energy !optimization !and !engineering, !3(3), !Pg.72-85, !DOI: !10.4018/ijeoe. !2014070105.

[4] Nayak !Nihar !Ranjan, !Mishra !Bikram !Keshari !, !Rath !Amiya !Kumar. !(2013), !A !time !efficient !clustering !algorithm !for !gray !scale !image !segmentation. !International !journal of !Computer !vision !and !image !processing, !3(1), !Pg.22-32

[5] Gharehchopogh !Farad !Soleimanian, !Ebrahimi !Samira !(2012). !A !Novel !approach !for !edge !detection !in !image !on !cellular !learning !automata. !International !journal of !Computer !vision !and !image !processing, !2(4), !Pg.51-61.

[6] Dixit !Karishma, !Jalal !Anand !Singh. !(2012), !A !Vision !based !approach !for ! Indian !sign !language !recognition. !International !journal of !Computer !vision !and !image !processing, !2(4), !Pg.25-36.

[7] Dubey !Shiv !Ram, !Jalal !Anand !Singh. !(2012), !Adapted !Approach !for !fruit !disease !identification !using !images. !International !journal of !Computer !vision !and !image !processing, !2(3), !Pg.45-58. !DOI: !10.4018 !/ijcvip. !2012070104.

[8] Win !Htwe !pa, !Khine !Phyo !Thu !Thu !, !Tun !Khin !New !Ni. !(2012). !A !structural !Analysis !based !feature !extraction !method !for !OCR !system !for !Myanmar !printed !document !images. !International !journal of !Computer !vision !and !image !processing, !2(1), !pg.16-41

[9] J. !Anitha, !C. Kezi !selva !Vijila !, !D. !Jude !Hemanth !(2010). !A !hybrid !genetic !algorithm !based !fuzzy !approach !for !abnormal !retinal !image !classification, !International !journal of !cognitive !Informatics !and !Natural !Intelligence,4(3),pg.29-43. !DOI:10.4018/jcini.2010070103.

[10] Krol !Dariusz, !Szlachetko !Boguslaw. !(2010).Automatic !Image !and !speech !recognition !based !on !neural !network. !Journal of !Information !Technology !Research, !3(2), !Pg.117

[11] Santo !Rafael do Espfrito, !Lopes !Roseli !de !Deus, !Rangayyan !Rangaraj !M. !(2009). !Classification of !breast !masses ! in !mammograms !using !radial !basis !functions !and !simulated !annealing, !International !journal of !cognitive !Informatics !and !Natural !Intelligence, !3(3), !pg.27-38.

[12] Yaremchuk !vanessa, !Dawson !Michael !R.W. !(2008), !Artificial !Neural !Network ! that !classify !musical !chords. !International !journal of !cognitive ! Informatics !and !Natural !Intelligence, !Volume-2, !Issue !3, !Pg.22-30

[13] Xu !Guangzhu, !Zhang !Zaifeng, !Ma !Yide.(2008).An !efficient !iris !recognition !system !based !on !intersecting !cortical !model !neural !network. IJCINI, !Volume !2, !Issue !2, !Pg.43-57.

[14] Xing !Weiwei, !Liu !Weibin, !Yuan !Baozong. !(2008).3D !Object Classification !Based !on !volumetric !parts. !International !journal of !cognitive ! Informatics !and !Natural !Intelligence, !2(1), !Pg-87-99.DOI:10.4018/jcini.2008010107

[15] Bruylants !Tim, !Munteanu !Adrian.(2015).Wavelet !based !volumetric !medical !image !compression,Elsevier,Volume31,Pg. !112-133. 